this week, and it deserves a broad audience.

The focus of the report is the collection of innovative (and, to some, controversial) accounts of the societal impact claimed for research. These impacts were reported as part of universities' submissions to the 2014 Research Excellence Framework (REF), which will be used to help to allocate public funds on the basis of merit (http://results.ref.ac.uk).

One university research group, for example, developed a model and database to quantify pollution of urban water sources. It also analysed peatland drainage systems to work out how to reduce water discolouration by dissolved organic carbon. The outputs of this research helped to improve water quality and the performance of the water industry.

Another group experimented on interventions that rewarded staff in primary health-care centres for their performance, to assess the effects on the quality of care delivery. The work delivered demonstrable impacts both nationally and internationally.

This week's report, The Nature, Scale and Beneficiaries of Research Impact, was produced by King's College London and Digital Science, a sister company of Nature's publishers. It features attractive diagrams of flows between research and impacts, but is heaped with qualifications as to its limitations: the analysis is based on text-mining of the roughly 7,000 submitted impact statements, which, at the time, were not searchable or classified with metadata.

Nevertheless, some of the report's broad conclusions are credible. UK academics make contributions that are felt in every country around the world, and have provided a rich variety of narratives about how universities affect policy, cultural development or research commercialization. Multiple fields have impacts on the same broad topics, yet each research field also splashes its impacts over multiple topics. Small institutions have larger societal impacts than is generally recognized.

The analysts freely admit that their report is a work in progress: the free-form nature of the REF meant that few impact statements presented information in standardized ways or used the same approaches to evaluate impact numerically. More consensus on approaches and standardization would probably be required to identify the qualitative and quantitative outcomes of research - yet a more prescriptive approach to gathering information might discourage academics from displaying their very diverse and heterogeneous impacts.

At this point, it is worth stepping back and noting the fundamental point. This exercise has shown that societal impacts can be documented and independently assessed.

Each of the assessment panels across academic disciplines has
"Applied research can gain its own professional merit and public recognition." documented the particular steps that it took to assess impact statements, and (assisted by a pilot study) to develop criteria for doing so (see go.nature.com/57wprj).

There will be justified debates about the cost of the exercise, how its outputs are geared to draw university funding, and the incentives to which such assessments will inevitably give rise. But there is a long-term perspective that should be considered.

In a country the size of the United Kingdom, no one should doubt that a significant proportion of the research base should be dedicated to research that tries to understand how the Universe and our planet function, and what makes humans tick. And outstanding performers in such basic research fully deserve the prestige given by prizes and academies.

But there are also outstanding performers in research addresses quite different goals: to directly improve the health, sustainability and resilience of individuals and the state of the world. Some of their work is published in highly specialized journals, but has a relatively broad societal impact.

By showing that standards of impact can be set and performance can be assessed, the REF has demonstrated in principle that applied research can gain its own professional merit and public recognition. Funders and institutions should celebrate, support and reward such achievements just as much as they celebrate prizes and academy memberships for fundamental research. Through such positive incentives and prestige, society at large will obtain a more appropriate balance of returns on its investments in university research.

\section{About time}

\section{The next few years will see NASA missions probe the innermost secrets of gas giants.}

$\mathrm{W}$ hen a powerful earthquake and tsunami destroyed large parts of Indonesia on 26 December 2004, Earth's crust was altered sufficiently to change the speed at which the planet rotates. Ever since, days have been 2.68 microseconds shorter.

But the rotations of other planets in the Solar System have been harder to determine. Saturn's, for instance, is still subject to vast uncertainties.

In Nature this week, three Israeli planetary scientists suggest that a day on Saturn is 15 minutes shorter than previously thought. It lasts for 10 hours, 32 minutes and 45 seconds, they say (R. Helled, E. Galanti and Y. Kaspi Nature http://dx.doi.org/10.1038/nature14278; 2015).

Because it is mostly a dense fluid of helium and hydrogen, Saturn would not be expected to have a single, well-defined rate of spin. But the planet is thought to have a rocky core, and to rotate roughly in unison in the same way that a solid object would. (The fluid Sun, by contrast, rotates $34 \%$ faster at its equator than at its poles.) Saturn's hazy atmosphere - the thickness of which is hard to pin down - makes it difficult to estimate how fast any firmer mass inside is spinning.

The two Voyager probes gave us the first modern estimate of Saturn's rotation period - around 10 hours and 39 minutes - some 35 years ago. They used radiofrequency emissions sent out by the planet's wobbling magnetic field. NASA's Cassini spacecraft, which has been orbiting Saturn since 2004, repeated those radio measurements but found a value that was not only substantially larger, but also changed with time. Experts began to question whether the technique, which had been applied successfully to Jupiter, was reliable for its smaller sibling Saturn.

Ravit Helled of Tel Aviv University and her colleagues took an approach that is, in a way, more traditional: they estimated the rotation indirectly, from the way it distorts the planet. Centrifugal forces give spinning celestial bodies a flattened shape, wider at the equator than at the poles. For Saturn, the effect is accentuated by the planet's size - centrifugal forces are stronger the farther one moves from the axis of rotation - and rapid spin. Moreover, its mostly fluid nature means that the planet deforms more easily. Not much is known about the distribution of mass inside Saturn, but something can be gleaned from its gravitational pull on objects that orbit it. Small deviations in Cassini's trajectories, for instance, have revealed that the gravitational field is not symmetrical.

This information is still not sufficient to nail down the internal structure of the planet or to calculate the rotation rate. So Helled and her co-authors did the next best thing. They produced a series of likely internal structures that narrow down the uncertainty to a 92 -second range.

Are they right? NASA plans to probe Saturn's gravitational field much more precisely towards the end of the Cassini mission, when the craft will dip into an elongated orbit and fly between the atmosphere and the system of rings. Just before its fuel runs out in 2017, Cassini will perform a controlled plunge into the planet. And the data it gathers along the way should help not just to elucidate the planet's make-up, but also to test models of how gas giants form in distant star systems. But Saturn
DNATURE.COM To comment online, click on Editorials at: go.nature.com/xhunqv will not be alone. From 2016, Juno, a major NASA mission that launched in 2011, will take similar measurements of the gravitational field of Jupiter. Sooner rather than later, it seems, these two gas giants will reveal their mysteries. 